anyone who uses it as a laboratory manual will recover its cost many times over. It is packed with solid data, drawn from the authors' own experience, and it is highly recommended as a truly valuable addition to peptide literature.

G. W. KenNer

\section{SOUND STUDIES}

\section{Ultrasonics}

Theory and Application. By G. L. Gooberman. Pp. xii + 210. (English Universities Press: London, January 1969.) $45 s$.

THE interest in ultrasonics has increased with its widening application in industry, oceanology and medicine, and tho output of books on the subject in recent years has correspondingly multiplied.

This latest volume is written by a physicist operating in an electrical engineering department, which should qualify him particularly well for the authorship. The first fifty pages of the book are devoted to the essential physical aspects of the subject and, although sparse in places, supply an adequate background for the later chapters. The importance of transducers in ultrasonic work is recognized by the amount of space devoted to their consideration and the author's own interest in high power' ultrasonics is shown to advantage in chapter five. Chapters six and seven will be of particular interest to physicists and chemists dealing with relaxation phenomena. One chapter is devoted to solids with a brief reference to such modern developments as spin waves, while general measurement techniques are briefly dealt with in another chapter.

Considering that the full title of the book is "Ultrasonics, Theory and Application", it is somewhat surprising that only some dozen pages are devoted to applications. Mention is made of Lamb waves, but Rayleigh surface waves are omitted somewhat surprisingly, having in mind their growing importance in industrial applications. Useful lists of references are included at the end of each chapter although they tend to omit some of the more recent work. The book is quite well produced, the errors appear to be few and the author has provided a useful basic book for intending workers in the field of ultrasonics.

R. W. B. Stephens

\section{FUNDAMENTAL DEFICIENCIES}

\section{Fundamentals of Mathematics from an Advanced Viewpoint}

By E. G. Kogbetliantz. Vols. 1 and 2: Algebra and Analysis-Evolution of the Number Concept and Determinants, Equations, Logarithms. Pp. xiv +547 . (Gordon and Breach: New York and London, 1968.) n.p.

THE introduction to this book, which ranges from clephants worshipping the Moon to the finite mind of man contemplating the infinite, promises a text of unusual and diverting content. In a sense one is not mistaken, for it includes a continual flow of delicious ancedotes. Unfortunately, no amount of rich historical detail can make up for the basic mathematical deficiencies which may be found.

Considering the length and title of the book, one might reasonably expcet a searching analysis of the concept of number, and yet the treatment is often vague and intuitive. A similar style pervades the second part on "Determinants, Equations and Logarithms" and the text is not really advanced, just occasionally obscure.

There are no exercises to help the reader, nor is there an index. The latter deficiency prevents one from tracing precise definitions in the text to find out exactly what the author is talking about. Possibly this is a calculated device because I am convinced that such precision is often non-existent.

For example, the author waxes loquacious on the concept of a limit and in an appendix criticizes the "usual definition", but he never really defines his terms. Instead he gives a quasi-mystical, pseudo-philosophical non-definition in terms of "infinitely small numbers". This idea could be explained in a suitable non-standard analysis framework but it proves positively misleading here. Precise definitions of continuity and convergence cannot be given and (as the author himself states) satisfactory proofs are often impossible. For example, the so-called "Rigorous Proof of Euler's Formula" (appendix 6) depends implicitly on the notion of continuity (a concept not discussed) and also on a non-proof of De Moivre's theorem given on page 220 .

The most insidious factor involved is that some nonproofs given may seem convincing to the unprepared beginner. This is because certain unstated subtle conditions cause the result to be true, although the author's proof applied in another case lacking these conditions could result in nonsense. For example, on page 398 the author blindly gives a proof that

$$
\lim _{n \rightarrow \infty}\left(1+\frac{1}{n}\right)^{n}=\sum_{m=0}^{\infty} m !
$$

which is equivalent to the statement $\lim \mathbf{a}_{m n}=\mathrm{a}_{m}$ implies

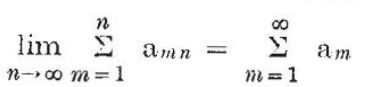

Should he care to apply this to the case $\mathrm{a}_{m n}=\frac{1}{n}$, he will find that $1=0$.

D. O. TALL

\section{ALGEBRAIC RINGS}

\section{Rings of Operators}

By Irving Kaplansky. (Mathematics Lecture Note Series.) Pp.vi+151. (Benjamin: New York and Amsterdam, 1968.) $\$ 3.95$.

THis book is a revision of lectures given by Irving Kaplansky at the University of Chicago more than ten years ago, bascd on his research into the foundations of the theory of von Neumann and related algebras expressed abstractly as operator rings. Their appearance in the present form is a tribute to the persistent interest in this field and to the work of the author. 'The theory is presented in an abstract form; indeed, it is very largely a tour de force in the handling of idempotent elements. It has roots, however, in the work of J. von Neumann on algebras of operators in Hilbert space and in continuous geometry. So, unlikely though it seems to be at first reading, the material has natural applications in theoretical physics.

The first chapter is on Baer rings, in which every annihilator ideal has an idempotent generator; these originated as endomorphism rings of geometric spaces. Even at this level of generality, the five basic sorts of order algebras make their appearance and are characterized by the behaviours of their sets of idempotents. We move nearer to the classical case with the introduction of Bacr-*rings, because an involution is superimposed. Here an equivalence, $e \sim f$, is set up between idempotents $e, f$ by supposing that $e, f$ generate isomorphic ideals. Then axioms $A$ to $F$ are imposed on this relation. The most important one, $F$, states that "if $\left\{e_{i}\right\}$ are orthogonal projections (idempotents) with least upper bound $e$; $\left\{f_{i}\right\}$ likewise with bound $f$, and $e f=0, e_{i} \sim f_{i}$ then $e \sim f^{\prime \prime}$ '. The main work of the book lies in removing the assumption $e f=0$. This result is easier to obtain in a purely infinite ring (no central finite idempotents), but the author needs additional axioms $G$ or $H$ in order to complete the other cases. All axioms hold in the classical examples. 